\title{
UTILIZAÇÃO DE BARREIRAS DE SEGURANÇA NO PREPARO DE DROGAS VASOATIVAS E SEDATIVOS/ANALGÉSICOS EM TERAPIA INTENSIVA PEDIÁTRICA*
}

\author{
Carla Susana Martinez Julca ${ }^{1}$, Patrícia Kuerten Rocha ${ }^{2}$, Andreia Tomazoni ${ }^{3}$, Bruna Figueiredo Manzo ${ }^{4}$, Sabrina \\ de Souza ${ }^{5}$, Jane Cristina Anders ${ }^{6}$
}

RESUMO: Objetivo: analisar a utilização de barreiras de segurança no preparo de drogas vasoativas e sedativos/ analgésicos. Método: estudo quantitativo, com 204 observações durante o preparo de medicamentos em uma Unidade de Terapia Intensiva Pediátrica no Hospital Pediátrico do Estado de Santa Catarina, de março de 2016 a maio de 2017. As barreiras analisadas foram: tipo de prescrição; transcrição da medicação, identificação do paciente e dados no rótulo; local de fixação; dupla checagem; preparo de infusões contínuas; interrupções. Os dados foram analisados por estatística descritiva. Resultados: as barreiras de segurança foram relacionadas à prescrição escrita (93,6\%); transcrição da medicação em rótulo (87,7\%); identificação do primeiro nome do paciente no rótulo (96\%); dados da medicação no rótulo sem a transcrição da via de administração $(99,4 \%)$; dupla checagem $(34,6 \%)$; interrupções durante o preparo $(52,9 \%)$. Conclusão: este estudo alerta a área sobre a necessidade da implementação de barreiras no intuito de uma prática clínica segura.

DESCRITORES: Segurança do paciente; Erros de medicação; Administração intravenosa; Sistemas de medicação no hospital; Enfermagem pediátrica.

\section{USE OF SAFETY BARRIERS IN THE PREPARATION OF VASOACTIVE DRUGS AND SEDATIVES/ANALGESICS IN PEDIATRIC INTENSIVE CARE}

\begin{abstract}
Objective: to analyze the use of safety barriers in the preparation of vasoactive drugs and sedatives/analgesics Method:quantitative study, with 204 observations during the preparation of medications in a Pediatric Intensive Care Unit in the Pediatric Hospital of the State of Santa Catarina, between March 2016 and May 2017. The barriers analyzed were: type of prescription; transcription of the medication, identification of the patient and data on the label; label attachment; double checking; preparation of continuous infusions; and interruptions. The data were analyzed using descriptive statistics. Results:the safety barriers were related to written prescription (93.6\%); transcription of the medication on the label $(87.7 \%)$; patient identification on the label only by the first name $(96 \%)$; data relating to the medication on the label failing to include the transcription of the route of administration $(99.4 \%)$; double checking (34.6\%); and interruptions during the preparation (52.9\%). Conclusion: this study alerts the area to the need to implement barriers so as to promote safe clinical practice.
\end{abstract}

DESCRIPTORS: Patient safety; Medication errors; Intravenous administration; Medication systems, hospital; Pediatric nursing.

\section{UTILIZACIÓN DE BARRERAS DE SEGURIDADEN LA PREPARACIÓN DE DROGAS VASOACTIVAS Y SEDANTES/ ANALGÉSICOS EN TERAPIA INTENSIVA PEDIÁTRICA}

RESUMEN: Objetivo: analizar el uso de barreras de seguridaden la preparación de drogas vasoactivas y sedantes/analgésicos. Método: estudio cuantitativo, con 204 observaciones durante la preparación de medicamentos en una Unidad de Terapia Intensiva Pediátrica en el Hospital Pediátrico del Estado de Santa Catarina, de marzo de 2016 a mayo de 2017. Las barreras analizadas fueron: tipo de prescripción; transcripción de la medicación, identificación del paciente y datos en el rótulo; local de fijación; doble verificación; preparación de infusiones continuas; interrupciones. Se hizo el análisis dedatos por medio de estadística descriptiva. Resultados: se asociaronlas barreras de seguridad a la prescripción escrita (93,6\%); transcripción de la medicación en rótulo (87,7\%); identificación del primer nombre del paciente en el rótulo (96\%); datos de la medicaciónen el rótulo sinla transcripción de la vía de administración $(99,4 \%)$; doble verificación (34,6\%); interrupciones durante lapreparación(52,9\%). Conclusión: este estudio hace un alerta sobre la necesidad de la implementación de barreras con el objetivo de llegar a una práctica clínica segura.

DESCRIPTORES: Seguridad del paciente; Errores de medicación; Administración intravenosa; Sistemas de medicaciónen el hospital; Enfermería pediátrica.

\footnotetext{
*Artigo extraído da dissertação de mestrado "Barreiras de segurança no preparo e na administração de medicamentos potencialmente perigosos em unidade de terapia intensiva pediátrica". Universidade Federal de Santa Catarina, 2016.
}

${ }^{1}$ Enfermeira. Mestre em Enfermagem. Universidade Federal de Santa Catarina. Florianópolis, SC, Brasil.

${ }^{2}$ Enfermeira. Doutora em Enfermagem. Docente de Enfermagem e da Pós-graduação da Universidade Federal de Santa Catarina. Florianópolis, SC, Brasil.

${ }^{3}$ Enfermeira. Doutoranda em Enfermagem. Enfermeira da Unidade de Terapia Intensiva Neonatal do Hospital de Clínica da Universidade Federal do Paraná. Florianópolis, SC, Brasil.

${ }^{4}$ Enfermeira. Doutora em Enfermagem. Docente de Enfermagem da Universidade Federal de Minas Gerais. Belo Horizonte, MG, Brasil.

${ }^{5}$ Enfermeira. Mestranda em Enfermagem. Enfermeira da Unidade de Terapia Intensiva Neonatal do Hospital de Clínica da Universidade Federal do Paraná. Florianópolis, SC, Brasil.

${ }^{6}$ Enfermeira. Doutora em Enfermagem. Docente de Enfermagem da Universidade Federal de Santa Catarina. Florianópolis, SC, Brasil. 


\section{INTRODUÇÃO}

As Unidades de Terapia Intensiva Pediátrica (UTIP) se caracterizam por serem locais com assistência de maior complexidade, permitindo a realização de terapias que incluem o uso de drogas vasoativas e sedativos/analgésicos ${ }^{(1)}$. As drogas vasoativas apresentam como principal função melhorar a perfusão tissular e fornecer oxigênio aos órgãos vitais ${ }^{(2)}$, enquanto os sedativos são utilizados para reduzir o desconforto e a ansiedade nos procedimentos realizados e diminuir a assincronia ventilatória durante o uso de ventilador mecânico ${ }^{(3)}$. Os analgésicos possuem a função de diminuição e prevenção da dor do paciente decorrente do estado clínico crítico $^{(4)}$.

Essas drogas são consideradas potencialmente perigosas e necessitam a aplicação de barreiras de segurança, devido ao risco de produzir dano irreversível ao paciente quando manipuladas de maneira inadequada ${ }^{(5)}$, principalmente na pediatria, pois a criança pode apresentar três vezes mais chance de sofrer danos quando comparada ao paciente adulto ${ }^{(6-7)}$.

Neste cenário, a fim de diminuir os erros, barreiras de segurança devem ser utilizadas durante o preparo de drogas vasoativas e sedativos. A equipe de enfermagem, responsável pelo preparo de tais drogas, deve implementar barreiras de segurança para diminuir as chances de cometer erros, providenciando assim assistência segura e de qualidade. As barreiras de segurança são definidas como um conjunto de medidas ou filtros que podem ser utilizadas pela equipe de saúde a fim de minimizar os riscos inerentes da assistência, evitando que um erro atinja e cause danos ao paciente ${ }^{(8-10)}$.

Como exemplos de barreiras de segurança no preparo de medicações, podemos citar a dupla checagem dos medicamentos, os nove certos na administração, protocolos no manejo de medicamentos potencialmente perigosos, protocolos de higienização das mãos, entre outros. Estas medidas diminuem a chance de cometer erros e promovem assistência mais segura ao paciente na utilização de medicações ${ }^{(11)}$.

Dessa maneira, inúmeras estratégias e barreiras de segurança podem ser aplicadas nos serviços de saúde a fim de diminuir os erros durante o processo de preparo de medicamentos. Contudo, observa-se que barreiras simples e que demonstram grande efetividade, como a identificação correta do paciente no preparo de medicações, não estão totalmente implantadas nas instituições ${ }^{(12)}$.

Especificamente, para prevenir erros durante o preparo de medicamentos potencialmente perigosos na pediatria, a The Joint Commission estabeleceu recomendações tendo como destaque a padronização da dosagem e a disponibilização de informação científica sobre a correta utilização destes medicamentos ${ }^{(13)}$. Além disso, a implementação de protocolos de segurança nos serviços de saúde sobre o manejo destas medicações também é uma das estratégias referenciadas pela Organização Mundial de Saúde (OMS) ${ }^{(14)}$.

Nesse contexto, uma estratégia adotada pelo Ministério de Saúde da Austrália consta de um profissional específico para a função de realizar o cálculo da dose das medicações nos serviços de saúde, a fim de detectar possíveis erros no processo de preparo e de administração dos medicamentos potencialmente perigosos ${ }^{(15-16)}$.

Considerando que o preparo de medicamentos potencialmente perigosos durante a hospitalização do paciente pediátrico faz parte da rotina terapêutica, a implementação de barreiras de segurança pode ser uma estratégia vantajosa neste processo garantindo a segurança do paciente.

Nesse sentido, devido à incipiente literatura nesta temática, é necessário explorar as barreiras de segurança que são utilizadas pelos profissionais de enfermagem durante a assistência nos serviços de saúde ${ }^{(17)}$. Portanto, o presente estudo tem como objetivo analisar as barreiras de segurança no preparo de drogas vasoativas e de sedativos/analgésicos em uma Unidade de Terapia Intensiva Pediátrica.

\section{MÉTODO}

Pesquisa quantitativa, descritiva exploratória, desenvolvida em uma UTIP, de um Hospital Pediátrico do Estado de Santa Catarina, no período de março de 2016 a maio de 2017. 
Para definição da população do estudo e cálculo da amostra, foi realizado um levantamento de registros das drogas vasoativas e sedativos/analgésicos preparados e administrados na UTIP, por um período de seis meses. Dessa maneira, obteve-se o registro de 66 drogas vasoativas e 240 sedativos/ analgésicos. O cálculo da amostra foi realizado tanto para as drogas vasoativas como para os sedativos/ analgésicos, por meio do programa estatístico SEstaNet ${ }^{\circledR}$ - UFSC, com nível de confiança de 95\%, resultando em uma amostra de 56 drogas vasoativas e 148 sedativos/analgésicos.

A coleta de dados constou de quatro etapas. A primeira foi a elaboração do instrumento de coleta de dados, baseado nas recomendações do American Society of Health Pharmacists ${ }^{(18)}$, Agência Nacional de Vigilância Sanitária ${ }^{(14)}$ e Institute for Safe Medication Practices ${ }^{(19)}$ sobre barreiras de segurança durante o processo de preparação e de administração de medicação. O instrumento foi divido em três blocos, sendo o primeiro constituído por características profissionais do participante, o segundo bloco pelas barreiras de segurança no preparo e o terceiro pelas barreiras de segurança na administração dos medicamentos.

Neste estudo, foram analisadas as seguintes barreiras de segurança: tipo de prescrição medicamentosa; transcrição da medicação no rótulo; identificação do paciente no rótulo; dados do rótulo (medicação, via, horário, dose e velocidade de infusão); local de fixação; dupla checagem; preparo de infusões contínuas; interrupções.

Na segunda etapa da coleta de dados, foi realizado o pré-teste do instrumento de coleta de dados por duas enfermeiras especialistas em Terapia Intensiva Pediátrica e no tema de segurança do paciente, a fim de aprimorar o instrumento.

Já na terceira etapa, foram realizadas reuniões com a chefia e a equipe de enfermagem da UTIP em questão, apresentado a proposta da pesquisa e o convite para a equipe participar. Por último, na quarta etapa foi realizada a observação aleatória na UTI, nos turnos da manhã e da tarde.

Os critérios de inclusão foram: profissionais que possuem experiência maior de três meses quanto ao preparo de medicamentos potencialmente perigosos; e como critério de exclusão, preparo de medicamentos potencialmente perigosos no momento de situações de emergência.

Para a realização do estudo, foi convidada a equipe de enfermagem da UTIP, dos 24 técnicos convidados 17 aceitaram participar do estudo, assinando voluntariamente o Termo de Consentimento Livre e Esclarecido. As enfermeiras convidadas afirmaram que o preparo de medicamentos fica direcionado aos técnicos de enfermagem, enquanto ao enfermeiro cabe a supervisão de tais ações. Assim, as enfermeiras convidadas não participaram do estudo.

Foram observados o preparo dos seguintes medicamentos: fentanil, midazolam, cetamina, dexmedetomidina, vercurônio, epinefrina, atropina, dobutamina, dopamina, noraepinefrina vasopressina, milrinona e amiodarona.

Os dados obtidos foram tabulados no programa Microsoft Exce ${ }^{\circledR} 2016$ e analisados por meio de estatística descritiva. O estudo seguiu os preceitos éticos da pesquisa e contou com a aprovação do Comitê de Ética que envolve pesquisa com seres humanos da instituição investigada, com número 1641960.

\section{RESULTADOS}

Participaram do estudo 17 técnicos de enfermagem, sendo 13 (76\%) do sexo feminino. A idade mínima dos participantes foi de 25 anos e a idade máxima de 50 anos. Destes, sete $(41 \%)$ trabalhavam na UTIP há entre cinco a dez anos; seis (35,2\%) trabalhavam há menos de cinco anos e quatro $(23,52 \%)$ há mais de 10 anos. Referente ao tempo de formação dos profissionais, obteve-se uma média de 10 anos.

Quanto à carga horária de trabalho, obteve-se uma média de 34 horas semanais. Com relação à formação adicional, três (17\%) profissionais possuíam ensino superior completo com especialização, quatro (23\%) ensino superior completo, dois (12\%) ensino superior incompleto e oito (47\%) não possuíam educação adicional. Destaca-se que nenhum destes profissionais recebeu capacitação 
quanto ao preparo de drogas vasoativas e de sedativos/analgésicos no último ano.

No estudo foram realizadas 204 observações durante o preparo de medicamentos potencialmente perigosos, dos quais $56(27,5 \%)$ corresponderam a drogas vasoativas e $148(72,5 \%)$ a sedativos/ analgésicos, conforme apresentado na Tabela 1.

Tabela 1 - Distribuição de frequências de medicações vasoativas e sedativos/analgésicos utilizadas em uma Unidade de Terapia Intensiva Pediátrica. Florianópolis, SC, Brasil, 2016

\begin{tabular}{lclc} 
Medicações vasoativas & $\mathbf{n ~ ( \% )}$ & Medicações sedativos/analgésicos & n (\%) \\
\hline Epinefrina & $27(48,2)$ & Midazolam & $56(37,8)$ \\
\hline Noraepinefrina & $9(16,7)$ & Fentanil & $50(33,7)$ \\
\hline Milrinona & $8(14,2)$ & Cetamina & $24(16,2)$ \\
\hline Dopamina & $5(8,9)$ & Dexmedotamidina & $10(6,7)$ \\
\hline Dobutamina & $4(7,1)$ & Fentanil+ Midazolam & $8(5,4)$
\end{tabular}

Em relação às barreiras de segurança no preparo de drogas vasoativas e sedativos/analgésicos, os resultados demonstraram que $13(6,4 \%)$ prescrições foram realizadas de forma verbal. Especificamente, as drogas vasoativas obtiveram uma $(1,8 \%)$ prescrição de forma verbal e $55(98,2 \%)$ foram prescritas de forma escrita. Já os sedativos/analgésicos tiveram 12 (8,1\%) prescrições verbais e 136 (91,9\%) prescrições escritas.

Quando analisada a transcrição da medicação em rótulo, verificou-se que a UTIP estabeleceu um rótulo impresso para ser preenchido com os dados do paciente e da medicação, devendo ser utilizado em todas as transcrições. Assim, a transcrição da medicação foi realizada em $179(87,7 \%)$ medicações, sendo 52 (92,8\%) relacionadas às drogas vasoativas e 127 (85,8\%) aos sedativos/analgésicos.

Ainda, a identificação do paciente no rótulo da medicação foi verificada com o nome completo da criança em seis $(3,4 \%)$ rótulos, o primeiro nome da criança em $172(96 \%)$ e o nome completo e apelido em um $(0,6 \%)$.

Em relação à identificação das medicações, dos 204 medicamentos preparados, 25 (12,2\%) não contaram com identificação, resultando em 179 (87,7\%) preparos de medicamentos identificados por meio do rótulo. Assim, durante a análise dos dados no rótulo quanto à identificação da medicação, via, horário, dose e velocidade de infusão, verificou-se que $178(99,4 \%)$ não transcreveram a via de administração (Tabela 2). Ainda, o rótulo de identificação das medicações foi fixado em 32 (17,3\%) destas, sendo que o local predominante de fixação foi na seringa $24(74 \%)$ e na bureta oito (25,8\%).

Tabela 2 - Identificação de medicações sem rótulo durante preparo de drogas vasoativas e de sedativos/ analgésicos em uma Unidade de Terapia Intensiva Pediátrica. Florianópolis, SC, Brasil, 2016

\begin{tabular}{lcc} 
Dados do rótulo & Sim $(\%)$ & Não $(\%)$ \\
\hline Nome da medicação & $179(100)$ & $0(0)$ \\
\hline Horário & $178(99,4)$ & $1(0,6)$ \\
\hline Via & $1(0,6)$ & $178(100)$ \\
\hline Dose & $179(100)$ & $0(0)$ \\
\hline Velocidade de infusão & $178(99,4)$ & $1(0,6)$
\end{tabular}


A dupla checagem no preparo dos medicamentos foi realizada 68 (34,6\%) vezes. Ademais, a medicação foi preparada com doses dobradas ou triplicadas em $80(39,4 \%)$ vezes, com a finalidade de aumentar a duração da infusão quando se tratava de infusão contínua.

Verificou-se que durante o preparo das medicações ocorreram 108 (52,9\%) interrupções aos profissionais, sendo que $64(59,2 \%)$ destas tiveram motivos associados à assistência (avaliação do paciente, alarme da bomba de infusão, parada cardiorrespiratória, exames complementares), e 22 $(20,4 \%)$ tiveram motivos pessoais (visualização do celular, conversa paralela). Quanto à quantidade de interrupções, 48 (44,4\%) observações foram interrompidas uma vez, sendo os técnicos de enfermagem os profissionais que mais interromperam durante o preparo de medicamentos, 46 (42,5\%) observações. Especificamente, as interrupções durante o preparo das drogas vasoativas ocorreram em 32 (57,1\%) observações e dos sedativos/analgésicos em 76 (51,3\%) (Tabela 3).

Tabela 3 - Interrupções durante o preparo de drogas vasoativas e sedativos/analgésicos em uma Unidade de Terapia Intensiva Pediátrica. Florianópolis, SC, Brasil, 2016

\begin{tabular}{|c|c|c|c|}
\hline Interrupções & & $\begin{array}{c}\text { Drogas vasoativas } \\
\mathbf{n}(\%) \\
\end{array}$ & $\begin{array}{c}\text { Sedativos/Analgésicos } \\
\text { n (\%) }\end{array}$ \\
\hline \multirow{4}{*}{ Número de vezes } & Uma vez & $17(53)$ & $31(40,7)$ \\
\hline & Duas vezes & $7(21,8)$ & $28(36,8)$ \\
\hline & Três vezes & $5(15,6)$ & $11(14,4)$ \\
\hline & Mais de três vezes & $3(9,3)$ & $6(7,8)$ \\
\hline \multirow{4}{*}{ Motivo das interrupções } & Assuntos pessoais & $5(15,6)$ & $17(22,3)$ \\
\hline & Ligações telefônicas & $7(21,8)$ & $5(6,5)$ \\
\hline & Alarmes de bombas de infusão & $4(12,5)$ & $6(7,8)$ \\
\hline & Motivos profissionais & $16(50)$ & $48(63,1)$ \\
\hline \multirow{5}{*}{ Profissional } & Técnico de enfermagem & $12(37,5)$ & $34(44,7)$ \\
\hline & Enfermeiro & $3(9,3)$ & $18(23,6)$ \\
\hline & Médico & $7(21,8)$ & $7(9,2)$ \\
\hline & Farmacêutico & $1(3,1)$ & $0(0)$ \\
\hline & Outros* & $5(15,6)$ & $8(10,5)$ \\
\hline
\end{tabular}

*Outros: fisioterapeuta, equipe de higienização, odontólogo.

\section{DISCUSSÃO}

O preparo de medicamentos pode ser um processo complexo e de risco, sendo que os resultados deste estudo demonstraram que apesar de algumas barreiras de segurança serem utilizadas pelos profissionais de enfermagem, não há uma institucionalização destas barreiras, pressupondo fragilidades na otimização e eficácia das mesmas ${ }^{(20)}$.

Dessa forma, a capacitação e o treinamento da equipe quanto ao manejo de medicamentos potencialmente perigosos é fundamental, a fim de potencializar assistência de qualidade e segura ${ }^{(21)}$. Ressalta-se que no último ano os profissionais de enfermagem não foram capacitados e treinados quanto às barreiras de segurança no manejo de tais medicamentos.

Estudo quantitativo realizado na Jordânia entrevistou 255 enfermeiros a fim de analisar os fatores individuais que influenciam os erros de medicação. Foi verificado que tais fatores foram principalmente a falta de treinamento e de capacitação dos enfermeiros quanto ao manejo correto dos medicamentos ${ }^{(22)}$.

Com relação à prescrição médica, verificou-se que a maioria foi realizada de forma escrita, porém ocorreram situações de prescrições verbais, as quais podem aumentar a chance de erros. Uma revisão integrativa de literatura realizada entre novembro de 2015 e fevereiro de 2017 tratou das estratégias, 
dos incidentes de segurança e etapas do processo medicamentoso na pediatria. Demonstrou que a prescrição médica é a etapa que mais apresenta eventos adversos durante o processo de preparo de medicamentos, uma vez que os pacientes pediátricos necessitam de cálculo de dosagem baseado no seu peso exato. Assim, sugerem como estratégia a implantação de prescrição médica eletrônica com sistema de alertas e protocolos para manejo de doses ${ }^{(23)}$.

Com relação à dupla checagem das medicações, foi verificado nos resultados que esta estratégia não apresenta padronização na unidade estudada e é realizada pela minoria dos profissionais. A dupla checagem consiste na conferência por dois profissionais de maneira simultânea e independente, a fim de identificar possíveis erros durante o preparo. Esta prática é recomendada para todos os serviços e principalmente no uso de medicamentos potencialmente perigosos, pois reduz significativamente os erros nesse processo ${ }^{(10)}$.

A transcrição da prescrição do medicamento em rótulo é uma barreira a ser realizada pela equipe de enfermagem, e possui como objetivo a preparação do medicamento seguindo as indicações da prescrição médica ${ }^{(24)}$. Verificou-se no estudo que a utilização desta barreira foi realizada na maioria das observações, no entanto há necessidade de padronizar sua prática no serviço da UTIP.

Estudo realizado em uma UTIP do Irã, entre 2013 e 2014, com objetivo de determinar a incidência de erros, analisou 512 doses de medicações. Estimou que os erros de transcrição apresentam 4.88 chances de ocorrer a cada 100 prescrições. Os erros ocorreram devido às informações transcritas erroneamente e foram relacionados à omissão, tempo errado, apresentação errada do medicamento e dose errada ${ }^{(25)}$.

Já estudo realizado no Uruguai, em unidades de internação pediátrica, com objetivo de identificar a prevalência de erros de prescrição e transcrição de medicamentos, analisou 276 prescrições e 448 transcrições realizadas pela equipe de enfermagem. Demonstrou a prevalência de erros de 66 para cada 100 transcrições. Os principais erros foram relacionados à dose de infusão 52 (11,7\%), dose incorreta 33 $(7,5 \%)$ e via incorreta $16(3,5 \%)^{(24)}$.

Quanto aos dados do paciente que são preenchidos no rótulo de preparo, é importante realizar identificação correta das informações da criança a fim de evitar falhas neste procedimento ${ }^{(20)}$. Também é recomendada a identificação correta das medicações que podem ocasionar danos ao paciente, principalmente com os medicamentos potencialmente perigosos ${ }^{(10)}$.

Cabe destacar que neste estudo o nome completo da criança foi transcrito em poucas ocasiões. No entanto, ressalta-se que a unidade é composta por um número pequeno de leitos e ainda, cada funcionário assume os cuidados de duas ou no máximo três crianças, diminuindo assim a chance de cometer falhas na identificação. Ademais, a transcrição do nome completo pode não ocorrer por falta da implementação de estratégias de segurança para identificação correta da criança.

Com relação à utilização do rótulo como barreira de segurança durante o preparo das medicações potencialmente perigosas, o estudo evidenciou que, entre os dados de identificação do medicamento no rótulo, a via correta nem sempre foi transcrita. Esta situação pode estar relacionada ao fato de que a UTIP conta com um rótulo pronto para ser preenchido com dados do paciente e medicação. Este rótulo foi desenvolvido pela equipe de enfermagem a fim de identificar o medicamento a ser preparado e possui espaços para preencher os dados do paciente, data, dose da infusão, horário de início, horário de término e nome do responsável do preparo do medicamento.

Estudo realizado em uma UTIP na Arábia Saudita, no ano de 2011, com objetivo de determinar a incidência e tipos de erros de prescrição, analisou 2.380 prontuários. Verificou que a taxa de erro foi de 56 para cada 100 medicações, sendo 525 (22,1\%) erros na dose e 285 (12\%) erros relacionados à via certa $^{(26)}$. Desse modo, ressalta-se a importância da implementação da via da medicação no rótulo de preparo de medicamento de medicamentos potencialmente perigosos a fim de prevenir enganos na administração ${ }^{(10)}$.

Ainda, a fixação do rótulo deve ser no dispositivo em que a medicação está armazenada, no intuito de atuar como barreira e evitar confusão quando há mais de uma medicação na bandeja ${ }^{(27,12)}$.

Destaca-se, que o preparo de medicamentos potencialmente perigosos para infusão contínua é uma das atividades comuns nas UTIP, devido à necessidade da criança receber tais medicamentos $\underline{\text { gradualmente e em doses exatas. Assim, o estudo demonstrou que as doses preparadas precisam ser }}$ 
duplicadas ou triplicadas a fim de disponibilizar o medicamento de maneira prolongada para 24 horas ou 36 horas. Entretanto, quando preparadas desta forma, há o risco de acontecer fold error, devido às interrupções mencionadas e a falta da dupla checagem ${ }^{(10)}$.

Com relação às interrupções durante o preparo dos medicamentos, estas situações podem ser consideradas de risco no preparo de medicamentos potencialmente perigosos. Estudo realizado na Turquia, com objetivo de identificar as interrupções durante a preparação e administração de medicamentos em unidades pediátrica, observou o preparo de 2.340 doses de 200 tipos de medicamentos e obteve como resultado 847 (36,1\%) interrupções. Destas, 616 (72,7\%) interrupções correspondem ao preparo; e foram principalmente causadas pelas mães das crianças $188(22,2 \%)$, médicos $151(17,8 \%)$ e enfermeiros $144(17 \%)^{(28)}$. Tais resultados corroboram com os achados nesta pesquisa, demonstrando que as interrupções realizadas durante a etapa de preparo de medicamentos podem produzir situações de erro.

Nesse sentido, é necessária a construção de um protocolo específico, a fim de padronizar o processo de preparo de medicamentos potencialmente perigosos ${ }^{(10)}$. Quando as barreiras de segurança são implementadas nas instituições, atingem-se resultados satisfatórios, como demonstrado em estudo quase experimental, cuja intervenção foi implementar o uso de um colete branco com o rótulo "Por favor não interromper" durante o preparo de 3714 medicamentos. Após a utilização desta medida, em duas semanas as interrupções diminuíram de 2355 para 1359; quanto à interrupção realizada pelos membros da equipe de saúde passaram de $773(32,8 \%)$ para $442(32,5 \%)$; quanto às interrupções por ligações telefônicas, passaram de 345 (14,6\%) para 136 (10\%) e perguntas quanto ao abastecimento de materiais de $57(2,4 \%)$ para $26(1,9 \%)^{(29)}$.

Portanto, as barreiras de segurança quando implementadas, devem ser trabalhadas com toda a equipe de saúde, a fim de potencializar os resultados esperados, promovendo maior segurança aos pacientes ${ }^{(30)}$.

A limitação deste estudo consiste em ser um estudo observacional onde, principalmente, nas primeiras observações a equipe poderia mudar seu comportamento habitual. Além disso, devido a uma questão de segurança da pesquisadora, não foi observado o período noturno, o que poderia complementar os dados encontrados.

\section{CONCLUSÃO}

A utilização das barreiras de segurança du rante o preparo de drogas vasoativas e sedativos/analgésicos em UTIP são estratégias que vem sendo realizadas pelo serviço, caracterizadas por medidas simples e que podem demonstrar diferença significativa na diminuição dos erros. No entanto, necessitam de padronização para aprimorar a eficácia e promover maior segurança ao paciente, como exemplo da prescrição escrita, utilização de rótulos específicos para transcrição de medicamentos que abordem as informações essenciais do paciente e do medicamento, a dupla checagem e a concentração do profissional durante o preparo das drogas, evitando interrupções no processo.

Portanto, há necessidade de fomentar mudanças institucionais para a padronização de barreiras de segurança, juntamente com o treinamento e capacitação dos profissionais, aliado à construção de um protocolo institucional quanto ao preparo e manejo dos medicamentos potencialmente perigosos.

Além disso, este estudo contribui para a área de Enfermagem evidenciando a necessidade de implementação de barreiras no intuito de se evitar erros no preparo e na administração de medicamentos potencialmente perigosos, o que poderia levar a morbidade ou mortalidade de pacientes. E deve ser publicado, pois evidencia como vem sendo realizada a prática de Enfermagem, indicando diretrizes para realizar uma assistência segura.

\section{- REFERÊNCIAS}

1. Doherty C, Donnell CM. Tenfold Medication Errors: 5 Years' Experience at a University-Affiliated Pediatric Hospital. Pediatrics. [Internet]. 2012 [acesso em 2017 dez 2017]; 129(5). Disponível em: http://dx.doi.org/10.1542/ 
peds.2011-2526.

2. Benham-Hermetz J, Lambert M, Stephens RCM. Cardiovascular failure, inotropes and vasopressors. Br. J. Hosp. Med. [Intenet]. 2012 [acesso em 2017 dez 19]; 73(Suppl5). Disponível em: https://doi.org/10.12968/hmed.2012.73. Sup5.C74.

3. Crego N. Pediatric sedation: Using secondary data to describe registered nurse practice in radiology. J. Radiol. Nurs. [Internet]. 2014 [acesso em 2017 dez 19]; 33(4). Disponível em: https://doi.org/10.1016/j.jradnu.2014.08.004.

4. Miranda ML, Bersot CDA, Villela NR. Sedação, analgesia e bloqueio neuromuscular na unidade de terapia intensiva. Revista HUPE. [Internet]. 2013 [acesso em 2017dez 19]; 12(3). Disponível em: https://doi.org/10.12957/ rhupe.2013.7537.

5. Institute for Safe Medication Practice (ISMP). List of High-Alert Medications in Acute Care Settings. [Internet]. EUA: ISMP; 2014[acesso em 2017 dez 19]. Disponível em: https://www.ismp.org/tools/highalertmedications.pdf.

6. The American Academy of Pediatrics. Principles of Pediatric Patient Safety: Reducing Harm Due to Medical Care. J. Pediatr. [Internet]. 2011[acesso em 2017 dez 19]; 127(6). Disponível em: https://doi.org/10.1542/peds.20110967.

7. Johnson PN, Miller JL, Hagemann TM. Sedation and Analgesia in Critically III Children. AACN Adv Crit Care. [Internet]. 2012 [acesso em 2017 dez 19]; 23(4). Disponível em: https://doi.org/10.1097/NCl.0b013e31826b4dea.

8. New Zealand Nurses Organisation. Guidelines for Nurses on the Administration of Medicines. [Internet]. New Zealand: New Zealand Nurses Organisation; 2012 [acesso em 2017 dez 19]. Disponível em: https://www. cdhb.health.nz/Hospitals-Services/Health-Professionals/IV-Link/Documents/NZNO\%20Guidelines\%20for\%20 nurses $\% 20$ on $\% 20$ the $\% 20$ administration\%20f\%20medicines.pdf.

9. The Joint Commission. The Nurse's Role in Medication Safety. SecondEdi. [Internet] USA: The Joint Commission; 2012 [acesso em 2017 dez 19]. Disponível em: https://doi.org/10.1097/01.NURSE.0000461850.24153.8b.

10. Instituto para Práticas Seguras no Uso de Medicamentos. Medicamentos potencialmente perigosos de uso hospitalar e ambulatorial: listas atualizadas de 2015. Bol. ISMP. [Internet]. 2015 [acesso em 2017 dez 19]; 4(3). Disponível em: http://www.ismp-brasil.org/site/wp-content/uploads/2015/12/V4N3.pdf.

11. Conselho Regional de Enfermagem de São Paulo (COREN-SP). Uso seguro de medicamentos: guia para preparo, administração e monitoramento. [Internet] São Paulo: COREN-SP; 2017 [acesso em 2017 dez 19]; 4(3). Disponível em: http://www.coren-sp.gov.br/sites/default/files/uso-seguro-medicamentos.pdf.

12. Souza S, Rocha PK, Cabral PFA, KusaharaDM. Use of safety strategies to identify children for drug administration. Acta Paul. Enferm. [Internet]. 2014 [acesso em 2017 dez 19]; 27(1). Disponível em: http://dx.doi.org/10.1590/19820194201400003.

13. The Joint Commission. Sentinel Event Alert. Joint Commission Perspectives. Preventing Pediatric. Medication errors [Internet]. 2008 [acesso em 2017 dez 19]; 39. Disponível em:https://www.jointcommission.org/assets/1/18/ SEA_39.PDF.

14. Agência Nacional de Vigilância Sanitária (ANVISA). Gerência de Vigilância e Monitoramento em Serviços de Saúde (GVIMS). Gerência Geral de Tecnologia em Serviços de Saúde (GGTES). Série Segurança do Paciente e Qualidade em Serviços de Saúde. Assistência segura: uma reflexão teórica aplicada à prática. [Internet] Brasília: ANVISA; 2013 [acesso em 2017 dez 19]. Disponível em: http://www.saude.pr.gov.br/arquivos/File/0SEGURANCA_ DO_PACIENTE/Modulo_1AssistenciaSegura.pdf.

15. Raban MZ, Lehnborn EC, Westbrook J. Evidence Briefings on Interventions to Improve Medication Safety. Interventions to reduce interruptions during medication preparation and administration. Centre for Health Systems and Safety Research Australian Institute of Health Innovation. [Internet]. 2013 [acesso em 2017 dez 19]; 1(4). Disponível em: https://www.safetyandquality.gov.au/wp-content/uploads/2013/12/Evidence-briefingson-interventions-to-improve-medication-safety-Reducing-interruptions-during-medication-preparation-andadministration-PDF-1.2MB.pdf.

16. Department Of Health Western Australia. WA Health High Risk Medication Policy: Office of Patient Safety and Clinical Quality. [Internet] Western Australia: Department Of Health Western Australia; 2014 [acesso em 2017 dez 19]. Disponível em: http://www.health.wa.gov.au/circularsnew/attachments/947.pdf. 
17. Belela ASC, Pedreira MDLG, Peterlini MAS. Erros de medicação em pediatria. Rev. bras. enferm. [Internet]. 2011[acesso em 2017 dez 19]; 64(3). Disponível: http://www.scielo.br/pdf/reben/v64n3/v64n3a22.pdf.

18. American Society Of Hospital Pharmacists. ASHP Guidelines on preventing medication errors in hospitals. Health-Syst. Pharm. [Internet]. 1993 [acesso em 2017 dez 19]; 50(2) Disponível em: http://www.ajhp.org/ content $/ 50 / 2 / 305$ ?sso-checked=tru.

19. Institute for Safe Medication Practice. Safe Practice Guidelines for Adult IV Push Medications: a compilation of safe practices from the ISMP Adult IV Push Medication Safety Summit. Institute for Safe Medication Practices Pennsylvania.[Internet] USA: Institute for Safe Medication Practice; 2015 [acesso em 2017 dez 19] Disponível em: http://www.ismp.org/Tools/guidelines/ivsummitpush/ivpushmedguidelines.pdf.

20. Rede Brasileira de Enfermagem e Segurança do Paciente. Estratégias para a segurança do paciente: Manual para Profissionais da Saúde. [Internet] Porto Alegre: REBRAENSP; 2013 [acesso em 2017 dez 19]. Disponível em: http://biblioteca.cofen.gov.br/wp-content/uploads/2017/10/Estrat\%C3\%A9gias-para-seguran\%C3\%A7a-dopaciente-manual-para-profissionais-da-sa\%C3\%BAde.pdf.

21. Conselho Regional de Enfermagem de São Paulo. Parecer Coren-SP 001/2014 -CT PRCI n 102.739/2012. Ementa: Possibilidade de recusa na administração de um medicamento (preparado/diluído) por outro profissional. Administração de medicamento (preparado/diluído) por profissional que não atua na área da saúde. São Paulo: Coren-SP; 2013.

22. Zyoud AH, Azimah N, Abdullah C. The Effect of Individual Factors on the Medication Error. Glob J Health Sci. [Internet]. 2016 [acesso em 2017 dez 19]; 8(12). Disponível em: http://dx.doi.org/10.5539/gjhs.v8n12p197.

23. Volpatto BM, Wegner W, Gerhardt LM, Pedro ENR, Cruz SS, Bandeira LE. Erros de medicação em pediatria e estratégias de prevenção: revisão integrativa. Cogitare enferm. [Internet]. 2017 [acesso em 2017 dez 19]; 1(22). Disponível em: https://revistas.ufpr.br/cogitare/article/view/45132/pdf .

24. Gutiérrez S, Mogni A, Berón A, Iramain R. Errores de medicación en niños hospitalizados. Arch Pediatr Urug. [Internet]. 2011 [acesso em 19 dez2017]; "'82(3). Disponível: http://www.scielo.edu.uy/pdf/adp/v82n3/v82n3a02. pdf.

25. Haghbin S, Shahsavari S, Vazin A. Medication Errors in a Pediatric Intensive Care Unit: Incidence, Types and Outcome. Trends in PhramaceuticalSciences. [Internet] 2016 [acesso em 19 dez 2017]; 2(2). Disponível em: http:// tips.sums.ac.ir/index.php/TiPS/article/view/68/77.

26. Al-Jeraisy MI, Alanazi MQ, Abolfotouh MA. Medication prescribing errors in a pediatric inpatient tertiary care setting in Saudi Arabia.BMC Research Notes. [Internet] 2011;4(1)[acesso em 19 dez 2017]. Disponível: https://doi. org/10.1186/1756-0500-4-294.

27. Australian Commission On Safety And Quality In Health Care (ACSQHC). National Safety and Quality Health Service Standards. [Internet] Sydney: ACSQHC; 2012 [acesso em 2017 dez 19]. Disponível em: https://www. safetyandquality.gov.au/wp-content/uploads/2011/09/NSQHS-Standards-Sept-2012.pdf.

28. Özkan S, Kocaman G, Öztürk C. Interruptions during pediatric medication preparation and administration. J Pediatr Res. [Internet]. 2016 [acesso em 2017 dez 19]; 3(2). Disponível em: https://doi.org/10.4274/jpr.98704

29. Currie L. Review: Reducing interruptions during medication administration: The White Vest study. Journal of Research in Nursing. [Internet]. 2014 [acesso em 2017 dez 19]; 19(3). Disponível em: https://doi. org/10.1177/1744987113484840.

30. Choo J, Johnston L, Manias E. Nurses' medication administration practices at two Singaporean acute care hospitals. Nursingand Health Sciences. [Internet]. 2013 [acesso em 2017 dez 19]; 15(1). Disponível em: https://doi. $\operatorname{org} / 10.1111 / \mathrm{j} .1442-2018.2012 .00706 . x$. 